


\title{
Robert Hutterer
}

\section{Das Paradigma \\ der Humanistischen Psychologie}

\author{
Entwicklung, Ideengeschichte \\ und Produktivität
}

SpringerWienNewYork 


\section{Ass.-Prof. Dr. Robert Hutterer}

Institut für Erziehungswissenschaften

Universität Wien

Wien, Österreich

Das Werk ist urheberrechtlich geschützt.

Die dadurch begründeten Rechte, insbesondere die der Übersetzung, des Nachdruckes, der Entnahme von Abbildungen, der Funksendung, der Wiedergabe auf photomechanischem oder ähnlichem Wege und der Speicherung in Datenverarbeitungsanlagen, bleiben, auch bei nur auszugsweiser Verwertung, vorbehalten.

(c) 1998 Springer-Verlag/Wien

Druck: Manz, A-1050 Wien

Graphisches Konzept: Ecke Bonk

Gedruckt auf säurefreiem, chlorfrei gebleichtem Papier - TCF

SPIN 10560052

Die Deutsche Bibliothek - CIP-Einheitsaufnahme

\section{Hutterer, Robert:}

Das Paradigma der humanistischen Psychologie : Entwicklung, Ideengeschichte und Produktivität / Robert Hutterer. - Wien ; New York : Springer, 1998 
Für Alexander, Alexander und Renate 


\section{Vorwort}

Dieses Buch ist das Ergebnis einer langen Auseinandersetzung mit einer Denkrichtung, die mit dem Etikett „Humanistische Psychologie“ nur ungenau bezeichnet ist. Denn sie berührt viele human- und sozialwissenschaftliche Problemfelder, die auch oder sogar speziell in anderen als der Psychologie beheimateten Fächern in dieser Nuancierung aufgegriffen werden. Die enge Verbindung dieser Denkrichtung mit der Bezeichnung „Psychologie“ scheint zumindestens zum Teil eine historisch nachvollziehbare Zufälligkeit zu sein. Ein Motiv, das mich dazu gebracht hat, dieses Buch zu schreiben, war die Unzufriedenheit mit der vorhandenen deutschsprachigen Literatur zu dieser Thematik. Oft wurde und wird die Humanistischen Psychologie mit bestimmten psychotherapeutischen Ansätzen gleichgesetzt. Oft erschöpfte sich die Darstellung der Humanistischen Psychologie in der Zusammenfassung von Ideen und Theorien einzelner ihrer Vertreter. Selten habe ich einen systematischen Anspruch bemerkt, der diese Denkrichtung von verschiedenen Seiten und Bezügen betrachtet, um ein verzweigtes Problembewußtsein, ein tieferes Verständnis für ihre Fragestellungen und auch ihre besondere Problematik zu vermitteln. Dieses hohe Ziel habe ich mir mit dieser Arbeit gesteckt. Der aufmerksame Leser erhält die Möglichkeit, die Texte und Zusammenhänge, in die die Entwicklung der Humanistischen Psychologie, ihre Ideen und ihre Produktivität eingebettet sind, nachzuvollziehen und die verschiedenen „roten Fäden“ mitzuverfolgen, die ein zusammenhängendes Verständnis dieser Denkrichtung erlauben.

Dieses Buch hätte ich nicht schreiben können, wäre ich nicht von vielen Seiten inspiriert und direkt oder indirekt unterstützt worden. Der Dank, den ich an dieser Stelle zum Ausdruck bringe, muß sich daher auch auf jene beziehen, die ihren Beitrag zu meinem Fortkom- 
men vielleicht nur schwer selbst erkennen können. Zuerst nenne ich hier meine Kolleginnen und Kollegen (Professoren und Mittelbau) am Institut für Erziehungswissenschaften der Universität Wien. Ich danke nicht nur jenen, die über die Jahre im akademischen Alltagsstreß gelegentlich Zeit für inhaltliche Diskussionen mit mir gefunden haben, sondern auch jenen, die aus der Distanz mir ein Beispiel für eigenständiges und unbequemes Denken gegeben haben. Die oft bedauerten Spannungen zwischen verschiedenen Positionen an diesem Institut waren auf inhaltlicher Ebene für mich eine Anregung. Ich danke besonders Professor Dr. R. Olechowski, Institutsvorstand und Leiter der Abteilung für Schulpädagogik und Allgemeine Didaktik. Er hat mir die Freiheit gewährt, meine eigene intellektuelle Entwicklung zu nehmen. Wer Verläufe akademischer Sozialisation kennt, wird mir beipflichten, daß dies nicht selbstverständlich ist. Nicht zuletzt danke ich auch jenen Studentinnen und Studenten, die in meinen Lehrveranstaltungen durch kluge Fragen und Beiträge mich zu klarerem Denken herausgefordert haben.

Einer weiteren Gruppe von Personen bin ich zu Dank verpflichtet. Als ich die Möglichkeit hatte, als Fulbright Scholar ein Semester an der West Georgia State University zu verbringen, erlebte ich eine amerikanische Version der Humanistischen Psychologie in Aktion. Der Dank gebührt dem Staff am dortigen Psychology Department, insbesondere Mike Arons, Christopher Aanstoos und Anne Richards. Sie ließen mir einen Einblick in ihre Forschung und Lehre und halfen mir dadurch, eine differenzierte Sichtweise über ihre Stärken und ihre Problematik zu entwickeln. Der Umstand, daß ich in ihrer Bibliothek deutschsprachige Werke von Spranger und anderen Autoren aus der deutschen und österreichischen Geschichte der Psychologie und Pädagogik fand, enttäuschte angenehm das Klischee vom amerikanischen Isolationismus. Ich fand dort ein akademisches Setting, das immer wieder für eine beträchtliche Auffrischung meiner Arbeitsmotivation sorgte.

Der Aufenthalt an der West Georgia State University war nicht mein erster direkter Kontakt mit der amerikanischen Humanistischen Psychologie. Als Student entstand mein Interesse an der Personenzentrierten Psychotherapie nach Carl Rogers zu einer Zeit, als die relevanten deutschsprachigen Veröffentlichungen dazu noch an den Fingern einer Hand abzuzählen waren. Ich hatte die Gelegenheit, die Entwicklung dieser Spielart der Humanistischen Psychologie im deutschen 
Sprachraum von Anfang an mitzuverfolgen und in bescheidenem Ausmaß in Publikationen und in meiner Tätigkeit als Psychotherapieausbilder auch mitzugestalten. Viele Anregungen für ein erweitertes Verständnis der Humanistischen Psychologie habe ich von meinen Mentoren und Kollegen erhalten. Besonders bedanke ich mich bei meinen Kollegen Dr. Gerhard Pawlowsky und Dr. Reinhold Stipsits für ihr langjähriges und beharrliches Bemühen, personenzentrierte und humanistische Tugenden der Offenheit, Toleranz und Achtsamkeit im intellektuellen Diskurs wie im kollegialem Umgang zu bewahren. Weiterer Dank gebührt meinen Mentoren Doug Land, Carl Rogers und in jüngster Zeit John Shlien. Doug Land hat mir über viele Jahre und in vielen professionellen und kollegialen Situationen das Beispiel eines praktizierenden Humanisten gegeben. Carl Rogers hat mir einen Weg aufgezeigt, eine einseitige Einstellung bezüglich wissenschaftlicher Forschung zu vermeiden und eine methodologische Offenheit zu entwickeln. Seine unbefangene Professionalität und seine unspektakuläre Wirksamkeit als psychotherapeutischer Praktiker wirft bis heute Fragen auf und hält mein Forschungsinteresse wach. Der direkte Kontakt zu ihm anläßlich gemeinsamer Seminare während zweier Wien-Aufenthalte hat mich vor der Idealisierung seiner Person und Position bewahrt, ohne den Respekt vor seinen Leistungen zu verlieren. Schließlich danke ich John Shlien für seine treffsichere, unmißverständliche und entschlossene Unterstützung. Ich hof$\mathrm{fe}, \mathrm{da}$ mir sein Mut und sein intellektueller Scharfsinn noch länger erhalten bleiben.

In der Reihe der Danksagungen möchte ich meine Familie nicht vergessen. Sie hat besonders in den letzten beiden Jahren in mir eine Person vorgefunden, die sich immer wieder zwischen Gedankenverlorenheit, Gereiztheit und Unansprechbarkeit bewegte. Ich danke meiner Frau und den Kindern für ihre Belastbarkeit, ihre Geduld und ihre Bereitschaft, sich auf mich einzustellen. Schließlich möchte ich mich bei Frau Margit Trummer für die sorgfältige Durchsicht und zügige Korrektur des Manuskripts herzlich bedanken. Ich habe durch ihre Arbeit wieder ein Stück der deutschen Sprache gelernt.

Robert Hutterer 


\section{Inhaltsverzeichnis}

Einleitung und Problemstellung - das Paradigma der Humanistischen Psychologie im Kontext seiner Entwicklung 1

\section{Der programmatische Kontext - die Erfolgsverheißung 11}

Die Bedeutungsvielfalt des Begriffs „humanistisch“ 12 Programmatische Aussagen 16 Humanistische Psychologie als „Dritte Kraft“ 24 Paradigmatische Unschärfen: Mehrdeutigkeiten und Entwicklungen 26

Heterogenität der Humanistischen Psychologie und allgemeine Wissenschaftsentwicklung $27 \cdot$ Humanistische Psychologie als sozial-aktivistische Bewegung und antiintellektualistische Tendenzen 29 - Protest, Offenheit und Interdisziplinarität 30 . Internationalität und eklektische Verbreitung $31 \cdot$ Transpersonale Psychologie: eine Abgrenzung 32 - New Age: eine Abgrenzung 34

\section{Der amerikanische Kontext - Pioniere, Immigranten} und Gegenkultur 39

Amerikanische Psychologie und das Aufkommen des Behaviorismus 39

Politische und ökonomische Bedingungen 42

Die amerikanische Psychologie der 20er und 30er Jahre 43 Immigration: der Import von Ideen 44

Die Immigration der Gestaltpsychologie 45 - Die Immigration der Psychoanalyse 47

Aufschwung der klinischen Psychologie in der Nachkriegs-Ära 54 
Nonkonformismus, Protest und Gegenkultur in den 60er Jahren 55

Die Institutionalisierung der Humanistischen Psychologie 56

Der Psychoboom und die Human-potential-Bewegung 60

3 Der europäische Kontext - Quellen, Wurzeln und Anregungen 69

Positivismus und naturwissenschaftlicher Fortschritt 70

Die Hinwendung zum Lebendigen: Kulturkritik und

Lebensphilosophie 71

Umdenken in den Wissenschaften: eine neue Epoche 74

Geisteswissenschaftliche Psychologie und Pädagogik, Gestalt- und

Ganzheitspsychologie 76

Die Reformpädagogische Bewegung 81

4 Der historische Kontext - Humanismen in der Geschichte 85

Der Begriff des Humanismus 85

Humanistische Ursprungsmotive in der Antike 87

Renaissance-Humanismus (1400 bis 1600) 90

Humanistische Motive der Aufklärung 95

Neuhumanismus (Mitte des 18. Jahrhunderts bis ca. 1830) 100

Der sogenannte Dritte Humanismus (erste Hälfte des

20. Jahrhunderts) 104

Die Humanismusdiskussion der Moderne 106

Kritik am Neuhumanismus 107 . Der gesellschaftskritische

Humanismus $110 \cdot$ Der existentialistische Humanismus 111

\section{Der philosophisch-anthropologische Kontext - Menschenbild} und Selbstinterpretation des Menschen 115

Humanismus als philosophische Herausforderung 115 Humanismus und Anthropologie 117

Funktionen von Menschenbildern 125

Zum Menschenbild der Humanistischen Psychologie 127

6 Der ideengeschichtliche Kontext - Verdichtungsprozeß vielfältiger Einflüsse 133

Behaviorismus 135

Psychoanalyse 139

Phänomenologie 143 
Existentialismus 152

Gestaltpsychologie und Feldtheorie 157

Organismische Theorie 162

Pragmatismus und Funktionalismus 175

Fernöstliche Philosophien 182

Persönlichkeitstheoretiker 186

Gruppenbewegung, methodischer Expressionismus und

Körperorientierung 196

Systemtheorie und moderne Naturwissenschaften 208

\section{Der methodologische Kontext - Wissenschaftsverständnis und Forschung 227}

Die methodologische Spannung 227

Wissenschaftskritik und Protest 229

Kritik gegen rigiden Objektivismus $230 \cdot$ Kritik am

Reduktionismus 231 - Kritik gegen Rigidität und

Selbstbeschränkung 232 • Problemorientierung versus

Methodenorientierung 233 - Entpersönlichung der Forschung:

Emotion, Werte und Wahrheit 234 - Entpersönlichung der Forschung:

Sterilität der Forschungs- und Publikationspraxis 235 .

Erkenntnispathologien: Psychologie der Wissenschaft und

Selbstreflexion 236

Die phänomenologische Tradition in der Humanistischen

Psychologie 238

Die Unvermeidlichkeit des bewußten Erlebens 240 - Die Funktion

der Phänomenologie $241 \cdot$ Strukturelle und funktionale

Phänomenologie 243

Das Methodenproblem der Humanistischen Psychologie 245

Methodologische Kontroversen innerhalb der Humanistischen

Psychologie 246 • Die methodologische Verletzbarkeit

der Humanistischen Psychologie 248

Perspektiven und Prinzipien humanistischer Forschung 252

Persönliches Wissen 259 - Authentische Wissenschaft 263 .

Wissenschaftsauffassung von Carl Rogers 268

Methodologische Vielfalt und Integration 282

Erweiterung methodologischer Perspektiven 283 .

Eine wissenschaftsphilosophische Perspektive: transzendentaler

Realismus und Forschung in geschlossenen und offenen Systemen 287 
Humanistische Psychologie als akademische Disziplin 298

Humanistische Psychologie in den Lehrbüchern und Fachzeitschriften 301

\section{Der thematische Kontext - Grundthemen und Produktivität} der Humanistischen Psychologie 305

Phänomenologie und Erleben 306

Psychologie der Bedeutung 307 - Subjektives Erleben 314

Holismus und Komplexität 314

Systemtheorie und Komplexität 316

Aktualisierungstendenz und Selbstaktualisierung 323

Hierarchie und relative Vormächtigkeit der Bedürfnisse 324 .

Aktualisierungstendenz und Selbst $328 \cdot$ Selbstverwirklichung und Selbstreflexion 335

Authentizität und psychische Gesundheit 340

Selbstaktualisierung und psychische Gesundheit 341 .

Die vollfunktionierende Persönlichkeit 343 · Selbstöffnung

und Erlebnisflüssigkeit 345

Organismus, Bewußtsein und Selbst 347

Der Organismus als Funktionsmatrix $348 \cdot$ Der Begriff

des Bewußtseins $352 \cdot$ Selbst 357

Dialog und Begegnung 362

Psychotherapie und Beziehung 365 - Begegnung und

Persönlichkeitsentwicklung in Gruppen $368 \cdot$ Vertrauensbildung in der Gruppe $370 \cdot$ Begegnung in der ersten Liebe 374

Selbsttranszendenz und Sinnfindung 377

Wille zum Sinn $380 \cdot$ Selbstbestimmung und Selbsthingabe 382

Autonomie, Werte und Wahlfreiheit 386

Bedürfnisse, Freiheit und Selbstreflexion in der Sozialisation 389 .

Freiheit und Nichtdirektivität als Entwicklungsfaktor 392

9 Der kritische Kontext - Humanistische Psychologie in der Kritik und Kontroverse 397

Das Zentrum der Kritik: Gegenkultur und Human-potential-

Movement 401

Kritik und Kontroversen zu Wissenschaftsentwicklung,

Metatheorie und Forschungsmethodologie 407 
Kritik und Kontroversen um das Konzept der

Selbstaktualisierung 412

Kritik und Kontroversen um die Bedeutung des Bösen und der menschlichen Destruktivität 420

Zusammenfassung und Ausblick 431

Literatur 445

Namenverzeichnis 473

Sachverzeichnis 480 